\title{
The assessment of minimal residual disease in chronic lymphocytic leukaemia: comparison of multi-colour flow cytometry and bone marrow trephine biopsy
}

\author{
E. Grey-Davies • A. Attygalle • A. Wotherspoon • \\ F. Carretero • A. Morilla $\cdot$ C. Dearden $\cdot$ E. Matutes
}

Received: 13 December 2011 / Accepted: 21 December 2011 / Published online: 17 January 2012

(C) Springer-Verlag 2012

\begin{abstract}
We investigated the value and degree of agreement between a sensitive four-colour flow cytometry (FC) method and bone marrow trephine (BMT) biopsy for detecting minimal residual disease (MRD) in 82 chronic lymphocytic leukaemia (CLL) cases. Concordance was $85 \%$, with $15 \%$ of cases discrepant (six BMT-positive/FC-negative and six BMT-negative/FC-positive cases). FC-positive/BMTnegative cases had a low-level MRD by FC $(0.05-0.9 \%)$, whereas BMT-positive/FC-negative cases had significant residual nodular disease. We conclude that $\mathrm{FC}$ and $\mathrm{BMT}$ biopsy are complementary investigations for MRD assessment in CLL, and both should be considered in the routine setting to assess MRD in CLL.
\end{abstract}

Keywords Minimal residual disease $\cdot$ Chronic lymphocytic leukaemia $\cdot$ Flow cytometry Bone marrow trephine biopsy

\section{Introduction}

The concept of minimal residual disease (MRD) in the context of chronic lymphocytic leukaemia (CLL) has emerged with improved therapy, and the goals are now moving from symptom control to eradication of all detectable disease. Recent advances, particularly the introduction of the monoclonal antibodies rituximab and alemtuzumab, have led to improved treatment responses and complete remission rates. Several groups have demonstrated that those patients who achieve an MRD-negative status have

E. Grey-Davies · A. Attygalle · A. Wotherspoon · F. Carretero $\cdot$

A. Morilla $\cdot$ C. Dearden $\cdot$ E. Matutes $(\square)$

Haemato-Oncology Unit, Royal Marsden Hospital,

203 Fulham Road,

London SW3 6JJ, UK

e-mail: estella.matutes@icr.ac.uk an increased progression-free survival and indeed that elimination of MRD is associated with an improved overall survival [1-3]. MRD assessment is particularly important after alemtuzumab therapy as eradication of MRD is associated with improved survival, whereas MRD-positive patients have similar outcomes to those who achieve a partial response [3]. Hence, there is a need for highly sensitive laboratory assessment of MRD.

MRD is defined as any disease detectable after treatment by the most sensitive technique available. A number of different methods can be employed for the assessment of MRD in CLL. Prior to 2000, the sensitivity of two-colour flow cytometry (FC) techniques was low. However the introduction of multi-colour FC has allowed sensitive and quantitative assessment of MRD [4]. An international standardised approach was proposed in 2007 to allow better comparison between clinical trials [4]. Approaches using allele-specific oligonucleotide polymerase chain reaction (PCR) to the immunoglobulin gene of the B cell are also highly sensitive techniques, with published guidelines on interpretation to allow standardisation of results between different laboratories [5]. Several studies have compared the sensitivity of MRD detection in CLL by multi-parameter FC and PCR techniques. Bottcher et al. demonstrated that FC and PCR are equally effective for MRD quantification in rituximabtreated CLL patients with a sensitivity of up to $10^{-4}$, whereas ASO IGH RQ-PCR is more sensitive for detecting MRD below that level [6].

Traditionally, response assessment in CLL has relied on bone marrow aspirate assessment, independent of biopsy findings $[7,8]$. There is minimal data to assess the role of BMT biopsy in the era of sensitive and quantitative techniques for MRD assessment as detailed above. In a study of 29 CLL patients, Maloum et al. demonstrated that BMT biopsy is a less sensitive technique for detecting MRD in CLL than multiparameter FC [9]. A comparison of 110 diagnostic follow-up 
Fig. 1 An FC-negative/BMpositive case demonstrating a large number of residual nodules associated with increased reticulin. Haematoxylin and eosin (a), reticulin stain (b) CD79a immunostain and (c) highlight multiple nodules of CLL

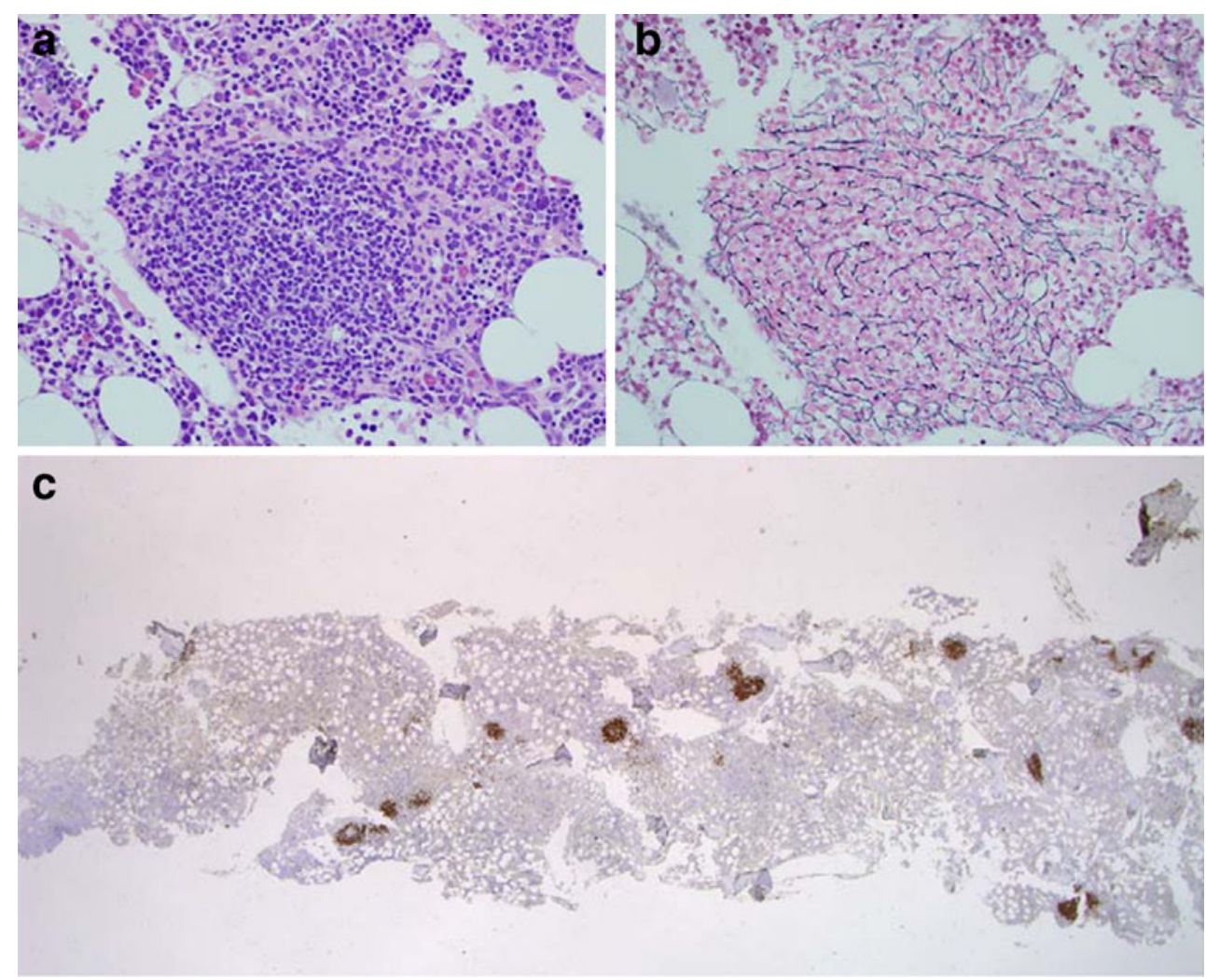

specimens after treatment for lymphomas (including $50 \mathrm{CLL}$ patients) analysed by dual-colour FC and BMT demonstrated consensus in $88 \%$ of the CLL cases [10]. Four biopsy negative samples had disease demonstrated by flow cytometry (at MRD level), whereas two samples were positive on the biopsy but negative by FC. In a study of 114 patients with nonselected B-cell lymphomas, there was consensus between FC and BMT biopsy assessment in $89.5 \%$ of cases [11].

This study aimed to compare the assessment of MRD by a sensitive four-colour FC method with the assessment of residual disease by BMT biopsy with immunohistochemistry in CLL patients.

\section{Materials and methods}

We retrospectively reviewed the bone marrow results of 82 consecutive patients who underwent assessment of MRD after treatment for CLL at the Royal Marsden Hospital between 2007 and 2010. We compared paired samples analysed by multi-parameter FC and by histological assessment of the BMT biopsy with immunohistochemistry. Flow cytometry was carried out using the international standardised approach [4] with antibodies targeted to CD5, CD19, CD20, CD38, CD22, CD81, CD79b, CD43, kappa and lambda (sensitivity $0.04 \%$ ). The bone marrow aspirate morphology was also reviewed, and haemodilute samples were excluded from the study. The nature of residual disease on
BMT biopsy was assessed by appropriate immunohistochemistry (CD20, CD79a, CD23, CD5 and CD3) to confirm the immunophenotype and to distinguish any nodular T-cell aggregates.

The patient group reflects our tertiary referral practice and comprised 49 male and 33 female patients with a median age of 61 years (range 36-87). A total of 43 patients had Binet stage $\mathrm{C}$ disease, 27 patients had progressive stage $\mathrm{B}$ and 12 patients had progressive stage A. They had received a range of different treatment regimens: fludarabine, cyclophosphamide and rituximab $(n=29)$, fludarabine and cyclophosphamide $(n=10)$, alemtuzumab $(n=12)$, alemtuzumab and methylprednisolone $(n=20)$, fludarabine, cyclophosphamide, mitoxantrone and rituximab $(n=2)$, chlorambucil $(n=2)$ or others $(n=7)$. Overall, $43 \%$ received rituximab therapy and $39 \%$ received alemtuzumab. Prior to commencing treatment, most patients had a heavily infiltrated bone marrow as demonstrated both by flow cytometry (median $75 \%$ of bone marrow leucocytes, range 3-96\%) and BMT (median $80 \%$ of total cellularity, range $23-95 \%$ ).

\section{Results}

The overall concordance between the two techniques was high, with 34 patients $(40 \%)$ achieving an MRD-negative status as assessed by FC and 34 patients $(40 \%)$ clear of residual disease as assessed in the BMT. However, there 
was a discrepancy between the results attained by $\mathrm{FC}$ and by BMT in $15 \%$ of cases. Of these, six cases were positive in the BMT but negative by FC and six cases were negative by BMT and positive by FC.

The six FC-positive/BMT-negative cases had low-level MRD as demonstrated by FC (median $0.075,0.05-0.9 \%$ of total bone marrow leucocytes). These patients had received FCR $(n=3)$, alemtuzumab $(n=1)$ or alemtuzumab and methylprednisolone $(n=2)$. In contrast, in all of the BMTpositive/FC-negative cases, there was a significant amount of residual nodular bone marrow CLL infiltrates, although all patients were in clinical remission. There was a median of 5.5 (range 2-18) residual nodules per trephine (median trephine length $13 \mathrm{~mm}$, range $12-15 \mathrm{~mm}$ ) and some were of considerable size (median maximal diameter $0.26 \mathrm{~mm}$, range 0.08 to $0.68 \mathrm{~mm}$ ). All six cases with residual disease demonstrated only on BMT had exclusively a nodular pattern of infiltrate, with a significant increase in reticulin within the nodule. These patients were treated with FCR $(n=4)$, FCMR $(n=1)$ and alemtuzumab and methylpredniolone $(n=1)$. Figure 1 illustrates an FC-negative/BMT-positive case with residual nodules associated with increased reticulin. Figure 2 illustrates an FC-positive/BMT-negative case with very low-level MRD demonstrated by four-colour FC.

Although the percentage of patients achieving an MRDnegative status was identical (40\%) when MRD was assessed by either FC or BMT, a further six patients had detectable residual disease when MRD was assessed using both techniques in combination as compared to either technique alone. Hence, the overall number of patients with no residual disease detected by either technique was $34 \%$ instead of $40 \%$. At the most recent follow-up, only two of the discrepant patients have progressed, both of whom were positive by FC and negative by BMT on MRD assessment. Due to the small number of cases and short follow-up, the clinical consequence of these discrepancies remains undetermined.

\section{Discussion}

Elimination of MRD in CLL is associated with improved overall and progression-free survival $[1-3,12]$. With more effective treatments available in CLL, the concept of MRD
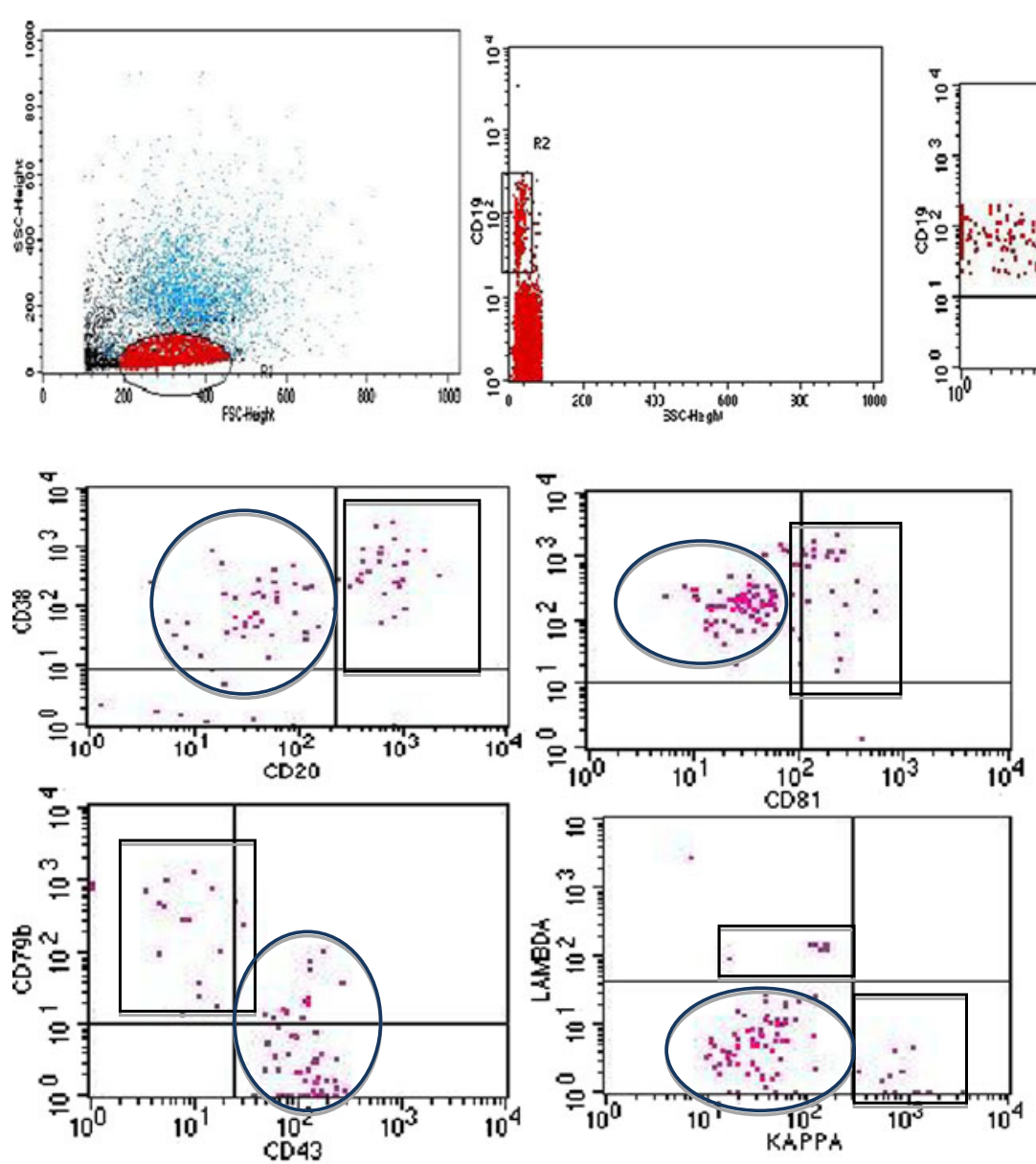

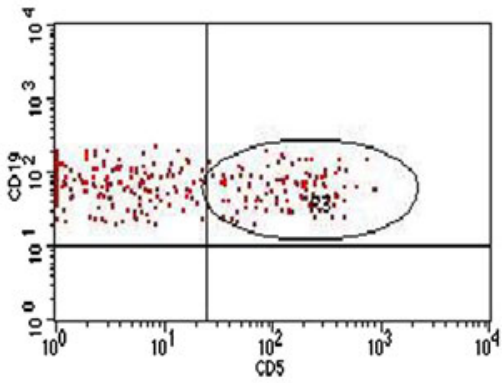

MRD positive case Residual CLL : $0.2 \%$ of total BM leucocytes.

The first 3 dot plots show sequential gating on lymphocytes, then CD19 positive lymphocytes and finally CD5/CD19 positive lymphocytes. The bottom 4 dot plots show the characteristic pattern of expression of residual CLL cells and how normal CD5+/CD19+ B cells can be distinguished from CLL cells.

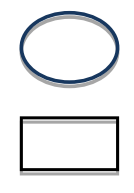

Shows residual CLL cells

Shows normal mature B cells

Fig. 2 An FC-positive/BMT-negative case with very low level MRD demonstrated by four-colour FC 
is taking on increased clinical importance. However, there is no consensus as to which is the most sensitive method to detect MRD or whether there is a need for the use of several techniques in a clinical setting. We have shown in this study an overall good consensus between FC and BMT biopsy for the assessment of MRD in CLL with agreement between the techniques in $85 \%$ of cases and discrepant results in the remaining. Those cases that were FC-positive/BMT-negative had low-level MRD as demonstrated by FC, confirming the utility of this highly sensitive technique. Such low levels of MRD may not be demonstrable on BMT biopsy when the residual leukaemic B cells are scattered in the interstitium, and demonstrating B-cell clonality by PCR or immunostaining for the Ig light chains is difficult in bone marrow sections. These cases confirm findings from previous studies suggesting that $\mathrm{FC}$ is a more sensitive technique than bone marrow trephine biopsy for demonstrating low-level disease $[9,10]$ but, as discussed later, it should not be taken in isolation.

A small proportion of cases only had MRD detected by histology, and in all six BMT-positive/FC-negative cases there was a significant amount of residual nodular disease which could be "missed" by relying solely on flow cytometry. The morphology of all aspirate samples was reviewed and it was confirmed that the samples analysed by flow cytometry were adequate in all the BMT-positive/FC-negative cases. This study clearly demonstrates the importance of performing a routine bone marrow trephine biopsy in addition to a bone marrow aspirate sample in CLL. All of the FC-negative/BMTpositive cases had a nodular pattern of residual disease associated with increased reticulin, which may prevent the successful aspiration of CLL cells. In general, when performing a bone marrow examination, the first, most particulate sample is generally used for morphological assessment and the subsequent samples for immunophenotyping, cytogenetic and molecular studies. This may reduce the sensitivity of detection of CLL cells in this type of specimen due to poor-quality, haemodilute samples. In this study, there is insufficient follow-up time to assess the clinical importance of the residual nodules. However, a previous study demonstrated that nodular PR in CLL represents MRD as confirmed by PCR [13].

We conclude that flow cytometry and bone marrow trephine biopsy are important complementary investigations for the assessment of MRD in CLL. In the era when MRD is taking on increased prognostic importance, it can be most sensitively assessed by using both techniques in combination.

Conflicts of interest The authors declare that they have no conflicts of interest.

\section{References}

1. Provan D, Bartlett-Pandite L, Zwicky C, Neuberg D, Maddocks A, Corradini $\mathrm{P}$ et al (1996) Eradication of polymerase chain reaction detectable chronic lymphocytic leukemia cells is associated with improved outcome after bone marrow transplantation. Blood 88:2228-2235

2. Bosch F, Ferrer A, Villamor N, González M, Briones J, GonzálezBarca E, Abella E, Gardella S, Escoda L, Pérez-Ceballos E, Asensi A, Sayas MJ, Font L, Altés A, Muntañola A, Bertazzoni P, Rozman M, Aymerich M, Giné E, Montserrat E (2008) Fludarabine, cyclophosphamide, and mitoxantrone as initial therapy of chronic lymphocytic leukemia: high response rate and disease eradication. Clin Cancer Res 14:155-161

3. Moreton P, Kennedy B, Lucas G, Leach M, Rassam SM, Haynes A et al (2005) Eradication of minimal residual disease in B-cell chronic lymphocytic leukemia after alemtuzumab therapy is associated with prolonged survival. J Clin Oncol 23:2971-2979

4. Rawstron AC, Villamor N, Ritgen M, Böttcher S, Ghia P, Zehnder JL, Lozanski G, Colomer D, Moreno C, Geuna M, Evans PA, Natkunam Y, Coutre SE, Avery ED, Rassenti LZ, Kipps TJ, Caligaris-Cappio F, Kneba M, Byrd JC, Hallek MJ, Montserrat E, Hillmen P (2007) International standardized approach for flow cytometric residual disease monitoring in chronic lymphocytic leukaemia. Leukemia 21:956-964

5. van der Velden VH, Cazzaniga G, Schrauder A, Hancock J, Bader P, Panzer-Grumayer ER, Flohr T, Sutton R, Cave H, Madsen HO, Cayuela JM, Trka J, Eckert C, Foroni L, Zur Stadt U, Beldjord K, Raff T, van der Schoot CE, van Dongen JJ (2007) Analysis of minimal residual disease by Ig/TCR gene rearrangements: guidelines for interpretation of real-time quantitative PCR data. Leukemia 21:604-611

6. Böttcher S, Stilgenbauer S, Busch R, Brüggemann M, Raff T, Pott C, Fischer K, Fingerle-Rowson G, Döhner H, Hallek M, Kneba M, Ritgen M (2009) Standardized MRD flow and ASO IGH RQ-PCR for MRD quantification in CLL patients after rituximab-containing immunochemotherapy: a comparative analysis. Leukemia 11:2007-2017

7. Hallek M, Cheson BD, Catovsky D, Caligaris-Cappio F, Dighiero G, Döhner H, Hillmen P, Keating MJ, Montserrat E, Rai KR, Kipps TJ (2008) Guidelines for the diagnosis and treatment of chronic lymphocytic leukemia: a report from the International Workshop on Chronic Lymphocytic Leukemia updating the National Cancer Institute-Working Group 1996 guidelines. Blood 111:5446-5456

8. Cheson BD, Bennett JM, Rai KR, Grever MR, Kay NE, Schiffer CA, Oken MM, Keating MJ, Boldt DH, Kempin SJ et al (1988) Guidelines for clinical protocols for chronic lymphocytic leukemia: recommendations of the National Cancer Institute-sponsored working group. Am J Hematol 3:152-163

9. Maloum K, Charlotte F, Divine M, Cazin B, Lesty C, Merle-Béral H, French Cooperative Group on CLL (2006) A comparison of the sensitivity of flow cytometry and bone marrow biopsy in the detection of minimal residual disease in chronic lymphocytic leukemia. Haematologica 91:860-861

10. Sah SP, Matutes E, Wotherspoon AC, Morilla R, Catovsky D (2003) A comparison of flow cytometry, bone marrow biopsy, and bone marrow aspirates in the detection of lymphoid infiltration in B cell disorders. J Clin Pathol 56:129-132

11. Carulli G, Canigiani S, Volpini M, Ciancia EM, Galimberti S, Zucca A, Marini A, Buda G, Petrini M (2005) Bone marrow infiltration in B-cell non-Hodgkin's lymphomas: comparison between flow cytometry and bone marrow biopsy. Recenti Prog Med 96:284-290 
12. Hallek M, Fischer K, Fingerle-Rowson G, Fink AM, Busch R, Mayer J, Hensel M, Hopfinger G, Hess G, von Grünhagen U, Bergmann M, Catalano J, Zinzani PL, Caligaris-Cappio F, Seymour JF, Berrebi A, Jäger U, Cazin B, Trneny M, Westermann A, Wendtner CM, Eichhorst BF, Staib P, Bühler A, Winkler D, Zenz T, Böttcher S, Ritgen M, Mendila M, Kneba M, Döhner H, Stilgenbauer S (2010) Addition of rituximab to fludarabine and cyclophosphamide in patients with chronic lymphocytic leukaemia: a randomised, openlabel, phase 3 trial. Lancet 376:1164-1174

13. Noy A, Verma R, Glenn M, Maslak P, Rahman ZU, Keenan JR, Weiss M, Filippa D, Zelenetz AD (2001) Clonotypic polymerase chain reaction confirms minimal residual disease in CLL nodular PR: results from a sequential treatment CLL protocol. Blood 97:1929-1936 